\title{
The Pattern of Menopausal Manifestations in a group of
}

\section{Egyptian Women}

Fatma A. Abo Romia ${ }^{a}$

\begin{abstract}
Background: Menopausal manifestations are affected by many ethnic, environmental and demographic factors. Aim of the study: to describe the pattern of menopausal manifestations in a group of Egyptian women. Subjects and Methods: The present study is a cross-sectional descriptive study. A sample of one hundred menopausal and premenopausal women in Tanta were selected to participate in the study. Based on review of relevant literature, structure interview was used to ask the studied women about their socio demographic characteristics and menopausal manifestations. Results: The studied women had a mean age of $(45.6 \pm 3.4)$ years, $75 \%$ of them were married. $20 \%$ one women were diabetics, $35 \%$ had hypertension and $60 \%$ had coronary artery disease. Three women were subjected to hysterectomy or oophrectomy. The age of menopause was 46 years in $52.0 \%$ of menopausal women. The most commonly reported symptom was bone aches (75\%) followed by sleep disturbance (71\%) and feeling anxious or nervous (65\%). Conclusions: the studied Egyptian women have a particular pattern that differs from other populations. Musculoskeletal manifestations and sleep disturbances are the most common manifestations in this group of Egyptian menopausal women.
\end{abstract}

Keywords: Menopausal symptoms -Egyptian pattern

\section{INTRODUCTION}

Menopause is a physiologic transition that takes place over several years. The earliest detectable hormonal change is a fall in ovarian secretion of inhibin, with a subsequent rise in follicle-stimulating hormone and maintained or increased levels of estradiol and finally loss of the menstrual cycle and the typical symptoms of menopause including increasing hot flushes and night sweats. ${ }^{(1)}$

Menopausal status of women was defined according to $\mathrm{WHO}$ definition. Women were considered to be in perimenopausal status if they were experiencing irregular menstrual bleeding within the last 12 months or amenorrhea

\footnotetext{
${ }^{a}$ Obstetric and Gynecologic Nursing, Faculty of Nursing, University of Tanta
} 
for more than 3 months but less than 12 months. Women experiencing amenorrhea for 12 consecutive months were considered to be in natural menopausal status. Women with a history of hysterectomy and/or oophorectomy were considered to be in surgical menopausal status. ${ }^{(2)}$

As women transit the peri-menopause, cycle irregularity increases, with the more frequent occurrence of prolonged ovulatory and anovulatory cycles. Levels of follicle-stimulating hormone and estradiol fluctuate increasingly and luteal function declines. Vasomotor symptoms tend to be most frequent around the time of final menses. The peri-menopause is thus a time of cycle and hormone variability and single hormone measurements provide little useful information, with the clinical history being the most appropriate method of assessing menopausal status. ${ }^{(3)}$

In general, many health problems have been attributed to menopause, but only vasomotor dysfunction and vaginal dryness are consistently associated with this time of life in epidemiological studies. Other common symptoms such as mood changes, sleep disturbances, urinary incontinence, cognitive changes, somatic complaints and sexual dysfunction may be secondary to other symptoms, or related to other causes. $^{(4)}$

Importantly, as hormonal changes during the menopausal transition directly or indirectly adversely affect quality of life, body composition and cardiovascular disease risk, maintenance of health parameters in the peri-menopausal years is crucial for a healthy post-menopause. ${ }^{(5)}$ In addition, it should be noted that women with more negative attitudes towards the menopause in general report more symptoms during the menopausal transition. $^{(6)}$

Aim of the study: the present study aims to describe the pattern of menopausal 
manifestations in a group of Egyptian women.

SUBJECTS AND METHOD:

Study design:

This is a cross-sectional descriptive study

\section{Study Setting}

The study was conducted at Maternal and Child Health Care Centers in Tanta city in Egypt. (Kohafa and Siger)

\section{Study sample:}

One hundred menopausal and perimenopasual women, fulfilling the chosen criteria (age between $40-60$ years and complaining of some somatic and psychosomatic complaints were selected to participate in the study. Exclusion criteria were women suffering from any malignancies, women with age $>60$ years and women suffering from clinically proved arthritis or previously diagnosed musculoskeletal problems (considering the fact that the symptoms presented of arthritis may sometimes be confused with those for osteoporosis).

\section{Data collection tool:}

An interview questionnaire sheet was developed by the researcher based on extensive review of relevant and recent literature. It was tested for content validity by 5 juries, who were experts in the related field for the purpose of the study.

Interviewing was conducted with the studied women to ask them about their socio-demographic characteristics and menopausal manifestations. To assure the clarity of the study questionnaire, the questions regarding the menopausal symptoms were rated using a Likert scale for clarity of each question, 1 meaning not clear at all and 5 meaning very clear. Body weight and height were measured and Body Mass Index (BMI) was calculated from the formula: body weight $(\mathrm{kg}) /$ height2 (m2). According to the World Health Organization definition, people with a BMI below $18.5 \mathrm{~kg} / \mathrm{m} 2$ tend to be underweight, and $25 \mathrm{~kg} / \mathrm{m} 2$ is normal , BMl over $25 \mathrm{~kg} / \mathrm{m} 2$ is defined as overweight and over $30 \mathrm{~kg} / \mathrm{m} 2$ 
as obese. A BMI between $18.5 \mathrm{~kg} / \mathrm{m} 2 .^{(7)}$

Pilot study:

Ten illiterate and ten literate women were chosen to perform a pilot study. The mean Likert scale for each question was calculated. Questions with mean Likert scale for clarity value lower than 4 were modified.

\section{Ethical considerations:}

Oral consent was taken from the women. Official permission clarifying the purpose of the study was obtained from the executive directors of health affairs department in Tanta to the concerned health centers to conduct the study and collect the necessary data.

\section{Statistical analysis}

Statistical analysis was carried out using the Statistical Package for Social Science 11.0 computer program (SPSS Inc., an IBM company, Chicago, IL, USA). Numerical data were represented in the form of mean $\pm S D$ while categorical data were represented in the form of number and percent

\section{RESULTS}

The socio-demographic characteristics of the studied women were shown in table1. The studied women had a mean age of $45.6 \pm 3.4$ years, $75.0 \%$ of them were married and $50.0 \%$ of them were obese. The associated co-moribidities in the studied women were shown in table-2. Twenty one women were diabetic, $35.0 \%$ had hypertension and 6 had coronary artery disease. Three women were subjected to hysterectomy or oophrectomy. Menopausal manifestations of the studied women are shown in table3. The age of menopause was 46 years in $52.0 \%$ of menopausal women. The most commonly reported symptom was bone aches $(75.0 \%)$ followed by sleep disturbance $(71.0 \%)$ and feeling anxious or nervous $(65.0 \%)$. 
Table. 1 Socio demographic characteristics of the studied women $(n=100)$

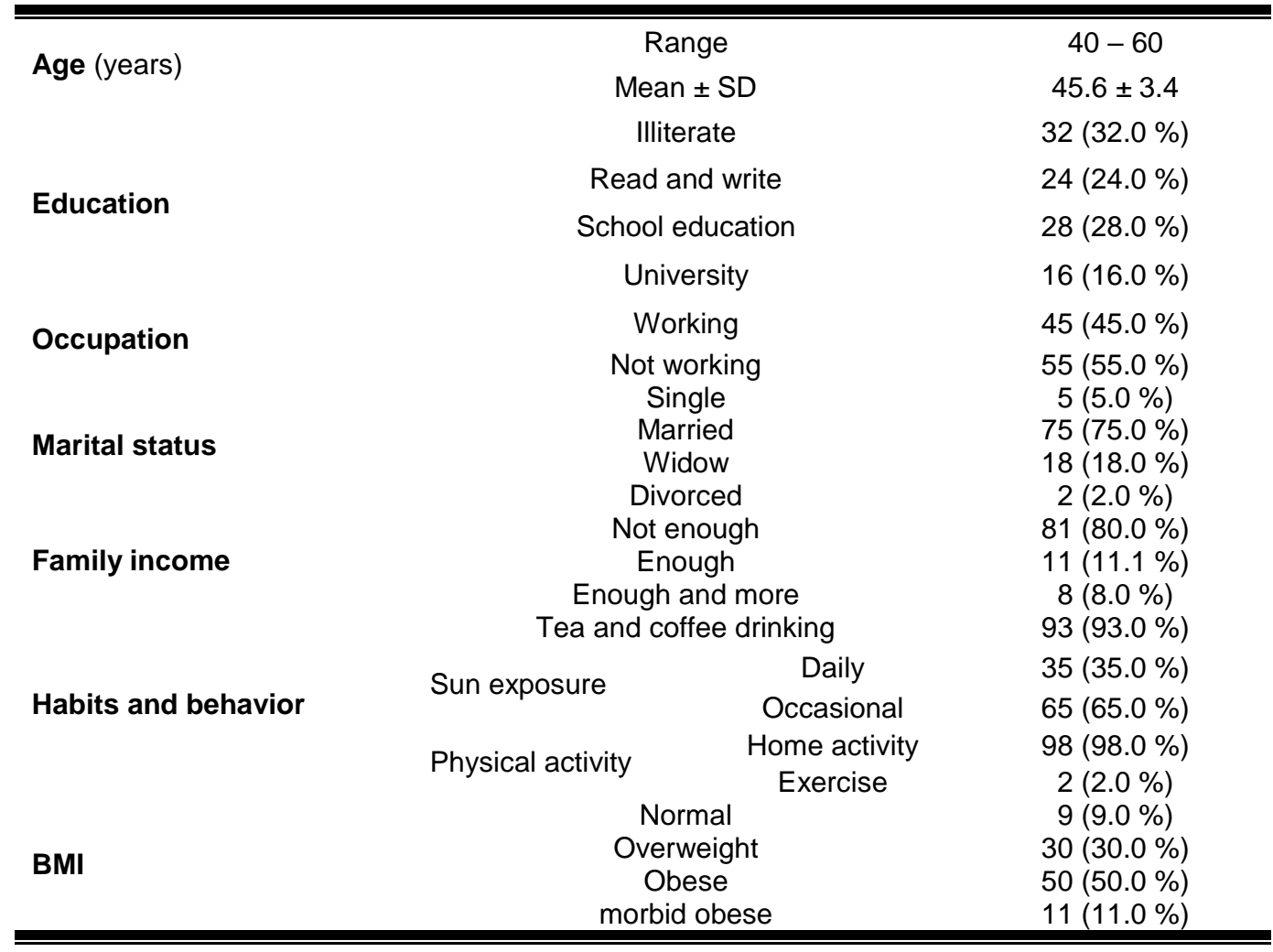

Table-2 Associated comorbidities in the studied women ( $n=100)$

\begin{tabular}{lcc}
\hline \hline & No & $\%$ \\
\cline { 2 - 3 } DM & 21 & 21.0 \\
Hypertension & 35 & 35.0 \\
Coronary heart disease & 6 & 6.0 \\
Hysterectomy or oophrectomy & 3 & 3.0 \\
\hline \hline
\end{tabular}


Table-3 Menopausal manifestations in the studied women $(n=100)$

\begin{tabular}{llcc}
\hline \hline & & No & $\%$ \\
\cline { 2 - 3 } Menstrual period regularity & Irregular & 70 & 70.0 \\
& No period for 6 months & 5 & 5.0 \\
& No period for $\geq 12$ months & 25 & 25.0 \\
& 46 y & 13 & 52.0 \\
Age of menopause* & 47 y & 3 & 12.0 \\
& 48 y & 6 & 24.0 \\
Fractures & $49 y$ & 3 & 12.0 \\
Bone aches & & 5 & 5.0 \\
Dental problems & & 75 & 75.0 \\
Hot flushes & & 37 & 37.0 \\
Palpitation & & 56 & 56.0 \\
Sleep disturbance & & 63 & 63.0 \\
& & 71 & 71.0 \\
Sexual desire ** & Unchanged & 37 & 49.3 \\
Dyspareunia ** & Increased & 7 & 9.3 \\
Vaginal dryness ** & Decreased & 31 & 41.3 \\
Vaginal itching & & 26 & 34.7 \\
Urinary incontinence & & 33 & 44.0 \\
Being dissatisfied with personal life & & 18 & 18.0 \\
Feeling anxious or nervous & & 10 & 10.0 \\
Experiencing poor memory & & 27 & 27.0 \\
Feeling depressed & & 65 & 65.0 \\
\hline \hline Menopausal women & & 58 & 58.0 \\
& & 56 & 56.0 \\
\hline
\end{tabular}

${ }^{*}$ Menopausal women $=25$

${ }^{* *}$ Married women $=75$ 


\section{DISCUSSION}

Menopause which is defined as urogenital atrophy, urinary tract infection and complete cessation of menstruation for incontinence, increased cardiovascular risk, twelve months or more is a normal physiological change experienced by middle age women. Some of menopausal symptoms experienced by these women can be severe enough to affect their normal daily activities. Unfortunately the majority of these women are not aware of the changes brought about by menopause. ${ }^{(8)}$

The menopause is a particularly important time in a woman's life. It is a time of adaptation and transition to a new biological situation which involves loss of the reproductive function. It is an aspect of human aging and a useful predictive risk marker of a variety of aging-related diseases and health problems. ${ }^{(9)}$

Several biological and psychosocial changes take place during this phase. Menopause may be associated with vasomotor symptoms, bone loss, somatic symptoms, sexual dysfunction and decreased libido, and loss of skin elasticity, which may have a significant negative impact on the overall quality of life of a substantial number of women. ${ }^{(10)}$

In the present study, the reported habits relevant to menopausal symptoms included tea and coffee drinking, occasional sun exposure and ordinary home activity. This is accordance with Karaçam and Abd Seke ${ }^{r(11)}$ who found that coffee consumption was associated with menopausal symptom score severity.

Regarding the relevant medical and surgical history, it was found that 21 women (21.0\%) had Diabetes Mellitus (DM), 35 women (35.0\%) had hypertension, 6 women $(6.0 \%)$ had coronary heart disease and 3 women $(3.0 \%)$ had hysterectomy or oophrectomy.

Comparatively, in the study of Pérez 
et al. ${ }^{(12)}, 36.6 \%$ of women had hypertension, $30.1 \%$ had low calcium intake, and $13.7 \%$ had DM.

Regarding the age of menopause, it was 46 years in 13 women (52\%), 47 years in 3 women, 48 years in 6 women $(24 \%)$ and 49 years in 3 women (12\%). However, the study of the Discigil et al. ${ }^{(13)}$ in Turkey showed that the distribution of age at menopause displays the highest peak at 45 years.

In the present study, the most commonly reported symptoms were bone aches $(75.0 \%)$ followed by sleep disturbance $(71.0 \%)$ and feeling anxious or nervous $(65.0 \%)$. However, in the study of Chim et al. ${ }^{(14)}$ in Singapore, the three most prevalent symptoms were low backache or aching muscles or joints (51.7\%), poor memory (45.1\%) and easy fatigability (38.8\%). Other reported symptoms included hot flushes (17.6\%), night sweats (8.9\%) and vaginal dryness (22.7\%).
In another study on Arab women in Australia, out of 29 symptoms, the most frequent symptoms were reported was 'feeling tired or worn out' (86\%), followed by 'aching in muscles and joints' (85\%). Sixty-three percent of the subjects reported 'hot flushes' and 54\% reported 'vaginal dryness during intercourse'. The least prevalent symptom was 'increased facial hair' $(35 \%)^{.(15)}$

In another study in Nigeria, joint and muscular discomfort was the most commonly reported symptom among all women in this study $(59.0 \%)$, followed by physical and mental exhaustion (43.0\%), sexual problems (40.4\%), and hot flushes $(39.0 \%){ }^{(16)}$ However menopause is a specialized phenomenon affected by the previous mentioned differences among people in deferent world areas

CONCLUSIONS: From the present study the following can be concluded:

The studied groups of Egyptian women have a particular pattern that differs from 
other populations. Musculoskeletal

manifestations and sleep disturbances are

the most common manifestations in

Egyptian menopausal women, followed by

anxiety and nervousness.

\section{RECOMMENDATIONS}

Based on the previous findings, the following can be suggested:-Health care especially Nurses should be aware about the fact that inspite of many menopausal manifestations are shared among all women worldwide, every population had a particular pattern of menopausal manifestations influenced by ethnic, environmental and demographic factors and the nurse back ground helps her in dealing with menopausal Clint . Further studies are recommended to investigate the probable determinants of menopausal manifestations in Egyptian women.

\section{REFERENCES}

1. Blake J. Menopause evidence-based practice. Best Pract Res Clin Obstet Gynaecol. 2006 Dec; 20(6):799-839.

2. Report of a WHO Scientific Group. Research on the menopause in the 1990s, WHO technical report series 866 . Geneva: World Health Organization; 1996.

3. Burger $H$, Woods NF, Dennerstein L, Alexander JL, Kotz K, Richardson G. :Nomenclature and endocrinology of menopause and perimenopause. Expert Rev Neurother. 2007 Nov; 7(11 Suppl):S35-43.

4. Nelson HD.: Menopause. Lancet. 2008 Mar 1; 371(9614):760-70.

5. Guthrie JR, Dennerstein L, Taffe JR, Lehert P, Burger HG.: The menopausal transition: a 9-year prospective population-based study. The Melbourne Women's Midlife Health Project. Climacteric. 2004 Dec; 7(4):375-89.

6. Ayers B, Forshaw M, Hunter MS.: The impact of attitudes towards the menopause on women's symptom experience: A systematic review. Maturitas. 2009 Nov 30.

7. Lobo RA, Kelsey J, Marcus R. Menopause: Biology and Pathobiology. San Diego: Academic Press, 2000.

8. Chedraui $P$, Blümel JE, Baron $G$, Belzares E, Bencosme A, Calle A, et al: Impaired quality of life among middle aged women: A multicentre Latin American study. Maturitas 2008, 61(4):323-9.

9. Bernis C, Sven Reher D. Environmental contexts of menopause in Spain: comparative results from recent research. Menopause 2007;4:777-87.

10. Utian WH. Psychosocial and socioeconomic burden of vasomotor symptoms in menopause: a comprehensive review. Health Qual Life Outcomes 2005; 3:47.

11. Karaçam Z, Seker SE. Factors associated with menopausal symptoms and their relationship with the quality of life among Turkish women. Maturitas. 2007 Sep 20;58(1):75-82.

12. Pérez JA, Garcia FC, Palacios S, Pérez M. Epidemiology of risk factors and 
symptoms associated with menopause in Spanish women. Maturitas. 2009 Jan 20;62(1):30-6.

13. Discigil G, Gemalmaz A, Tekin N, Basak O. Profile of menopausal women in west Anatolian rural region sample. Maturitas. 2006 Oct 20;55(3):247-54.

14. Chim H, Tan BH, Ang CC, Chew EM, Chong YS, Saw SM. The prevalence of menopausal symptoms in a community in
Singapore. Maturitas. 2002 Apr 25;41(4):275-82.

15. Lu J, Liu J, Eden J. The experience of menopausal symptoms by Arabic women in Sydney. Climacteric. 2007 Feb; 10(1):72-9.

16. Olaolorun FM, Lawoyin TO. Experience of menopausal symptoms by women in an urban community in Ibadan, Nigeria. Menopause. 2009 Jul-Aug;16(4):822-30. 